\title{
$N 12.25380$
}

REMOTE SENSING OF WINDS AND ATMOSPHERIC

TURBULENCE BY CROSS-CORRELATION OF

PASSIVE OPTICAL SIGNALS

A. J. Montgomery

IIT Research Institute

Chicago, Illinois 60616

\section{ABSTRACT}

This paper describes a new method

for the remote measurement of winds

and atmospheric turbulence by the cross-correlation of passive optical

signals. If small local variations in atmospheric density, temperature or other parameters cause fluctuations in scattered or thermal radiation detected by a radiometer on the ground, then the cross-correlation of the fluctuations detected by two radiometers with crossed fields of view can yield turbulence information pertaining to the region about this intersection point. When the fields of view are not quite crossed turbulent eddies will be convected through the fields of view sequentially, and the transit times of the eddies identified by the correlation procedure will yield wind information.

The successful application of this technique, detecting fluctuations in scattered sunlight, has demonstrated both the potential, and the present limitations of the method, which are discussed in this paper. Results for the power spectrum of the fluctuations and for winds at an altitude of 61 
meters are shown, and the wind measurements are compared to similar measurements made with a standard anemometer located on top of a 61 meter tower.

\section{INTRODUCTION}

The determination of wind profiles, turbulence scales and three dimensional wave-number components is of great importance in numerical methods of weather prediction and in clear air turbulence studies. Some type of remote sensing system is quite clearly required if this information is to be obtained conveniently. In the case of wind measurements which are of particular importance in weather prediction for the tropical and subtropical regions of the earth, measurements at a sufficient number of grid points over a time interval of a few hours could be obtained only with a satellite remote sensing system.

At this time no proven technique is available by which wind fields may be remotely measured. However, a limited amount of wind field information may be inferred from some of the atmospheric parameters measured with present $1 y$ orbiting meteorological satelities. This problem is discussed by W. Nordberg in COSPAR Transactions No. 3, "Status Report on the Applications of Space Technology to the World Weather Watch."

This paper describes a new technique presently under development which shows promise for the remote detection of winds directly. Wind components have been successfully determined at 200 and 400 foot altitudes from the crosscorrelation of output signals of two photometers located on the ground with their fields of view intersecting at these altitudes. In these tests scattered sunlight was detected; however, extension of the technique to infrared measurements of water vapor, ozone or other possible atmospheric tracers is presently being studied.

This technique was first suggested by M. J. Fisher (1964) and has been developed by IIT Research Institute and NASA, Marshall Space Flight Center, for the measurement of convection speeds and turbulent flow properties in fluid flows. Since the power of the technique has very clearly been demonstrated in measurements of convection speeds, eddy scales, and eddy lifetimes, in turbulent shear layers produced by an air jet, 
(Fisher and Krause, 1967), the basic method will be described with reference to these successful aerodynamic tests.

In the course of this paper we shall discuss:

(1) The basic concept of the crossed-beam technique

(2) Successful application to subsonic and supersonic aerodynamic flows

(3) Methods of applying the technique to the atmosphere

(4) Source of atmospheric fluctuations

(5) Particular problems associated with the technique due to the unstationary nature of atmospheric phenomena

(6) Initial measurements of winds and their comparison with data from meteorological towers

(7) Some future developments which are currently being considered.

\section{CROSSED-BEAM TECHNIQUE}

The crossed-beam experimental configuration as used in the aerodynamic test program is shown in Figure 1. Two collimated

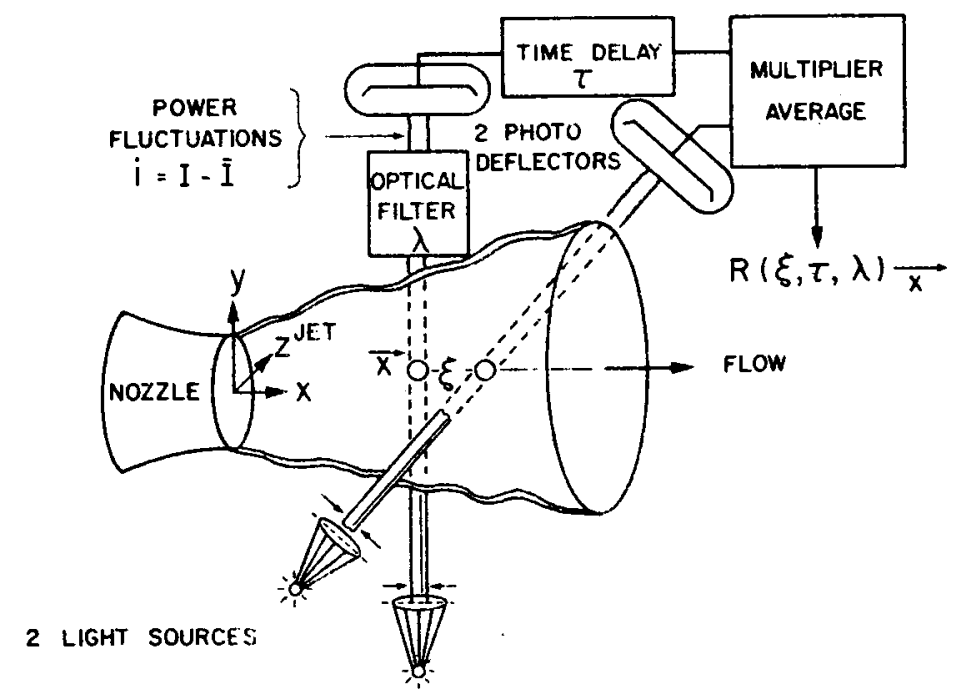

Figure 1. Space Fixed Crossed Beam Test Arrangements 
beams from the two light sources shown intersect $(\xi=0)$ at the point of interest in the flow. The spectral content of the radiation that is detected is selected by the optical filter or monochromator which precedes the detector in both beams, and the wavelength has to be chosen so that there are absorption or scattering losses from the beam during its passage through the flow. Since the flow is turbulent there will be variations in the numbers of absorbers or scattering agents along the two beams which will cause fluctuations in the detected signals. Measurement of the cross-correlation between the signals from the two detectors will yield information about the fluctuation level in the region of intersection of the two beams in the following manner. If a turbulent eddy having associated with it a higher or lower concentration of scatterers or absorbers than the surrounding medium passes through one of the beams it will cause fluctuations in the detected signal. Only if it passes through intersection point of the two beams will a zero time delay correlated fluctuation be produced. Thus it may be shown that the output of the correlator depends only on the properties of those eddies which pass through the beam intersection point. The correlation coefficient, which is normalized with respect to the uncorrelated fluctuations; depends as well on the eddy scales and the way in which the strength of the fluctuations in absorber or scatterer concentration vary along the two beams. Let us now consider one beam displaced a distance $\xi$ down-stream from the other beam. An eddy convected along the line of minimum separation between the beams will cause fluctuations at one detector and a correlated fluctuation at the second detector a time $\tau$ later, where $\tau$ is the time for the eddy to be convected the distance $\xi$. Thus if the correlation between the two signals is measured as a function of time delay, this correlation function will be a maximum for $t=\tau$.

To put this discussion on a more formal footing, the mathematical basis of the technique will be briefly considered. Using the coordinate system shown in Figure 1, the point of beam intersection has coordinates $(x, y, z)$, where the $y$ and $z$ axis are orientated along the directions of the crossed beams. Distances from the point of intersection are denoted by $\xi$, $\eta$ and $\zeta$ in the $x, y$, and $z$ directions, respectively: The intensity measured by the y-axis detecting system will be given by:

$$
I_{1}(t)=I_{0} \exp \left\{-\int \mathrm{K}(\mathrm{x}, \mathrm{y}+\eta, \mathrm{z}, \mathrm{t}, \lambda) \mathrm{d} \eta\right\}
$$

where $I_{Q}$ is the initial beam intensity and $K$ is the extinction coefficient. The reduction in the intensity of the beam will result from both absorption and scattering losses. In a completely general case both scattering into the field of view of the detecting system and emission from within the field of 
view have to be considered and, in fact, are of vital importance in the application of the crossed-beam technique to atmospheric measurements. However, the simple theory, only, will be considered here.

If the extinction coefficient, $K$, is divided into a mean and time varying part, equation (i) can be written

$I_{1}(t)=I_{0} \exp \left\{-\int\langle k(x, y+\eta, z)\rangle d \eta \exp \left\{-\int k(x, y+\eta, z, t) d \eta\right\}\right.$

Since the second integral in this expression will be, or can be made to be, -very much less than unity, a linear expansion can be used, and the result obtained for the fluctuating signal at the detector is

$$
i_{1}(t)=-\left\langle I_{1}\right\rangle \int k(x, y+\eta, z, t) d \eta
$$

The signal at the second detector will be of similar form and the covariance of the two detector signals will be given by

$$
G(x, y, z) \equiv 1 / T \int_{0}^{T} i_{1}(t) i_{2}(t) d t
$$

where $T$ is the period of integration which ideally is of sufficient length to yield a statistically stationary value of $G(x, y, z)$. Thus substituting for $i_{1}(t)$ and $i_{2}$. $(t)$ we $c a b$ write for the covariance of the signats that

$$
\begin{aligned}
\mathrm{G}(\mathrm{x}, \mathrm{y}, \mathrm{z})= & \left\langle\mathrm{I}_{1}\right\rangle\left\langle\mathrm{I}_{2}\right\rangle \int_{r_{i}} \iint_{\zeta} 1 / \mathrm{T} \int_{\mathrm{o}}^{\mathrm{T}} \mathrm{k}(\mathrm{x}, \mathrm{y}+\eta, \mathrm{z}, \mathrm{t}) \\
& \mathrm{k}(\mathrm{x}, \mathrm{y}, \mathrm{z}+\zeta, \mathrm{t}) \operatorname{dtd} \zeta \mathrm{d} \eta
\end{aligned}
$$

The term inside the time integral is clearly the covariance of the fluctuations in extinction coefficient at the points ( $x$, $y+\eta, z)$ and $x, y, z+\zeta$ ) which will be significantly different from zero only within the correlated area about the beam intersection point. If the assumption is made that the fluctuations in the $\mathrm{k}$ do not vary appreciably over this correlation area then the covariance of the two detector signals can be written

$$
G(x, y, z)=\left\langle I_{1}\right\rangle\left\langle I_{2}\right\rangle \overline{k^{2}(x, y, z, t)} L_{y} L_{z}
$$

where $\mathrm{L}_{\mathrm{y}}$ and $\mathrm{L}_{\mathrm{z}}$ are the integral length scales in the $\mathrm{y}$ and $\mathrm{z}$ directions, respectively. This measured quantity is therefore related directly to the local turbulent intensities and the integral scales at the point of intersection of the beams. 
A point to note here is that the spatial resolution is determined in theory by the beam diameters and that spatial resolutions obtained in turbulent intensity measurements are considerably less than the integral scale of the turbulence.

Other turbulent properties may be obtained from measurements of space-time correlations. In Figure 1 the second beam is displaced a distance $\xi$ in the flow direction and the covariance of the two detector signals can be measured for different beam separation time delays $\tau$. Fisher and Krause have shown that, to a useful degree of approximation, the space time correlation coefficient that would be measured by two point probes located at the points $A \& B$ can be measured with the crossed-beam system,

$$
r(\xi, \tau)=G(x+\xi, y, z, \tau) / G(x, y, z) .
$$

By measuring this space-time correlation coefficient over range of $\xi$ and $\tau$ such properties of interest as integral length scales, turbulent spectra, convection velocities, moving axis time scales and eddy lifetimes can be obtained.

This can most easily be understood with reference to some of the experimental results that have been obtained, as shown in Figure 2. Each of the individual curves is the correlation

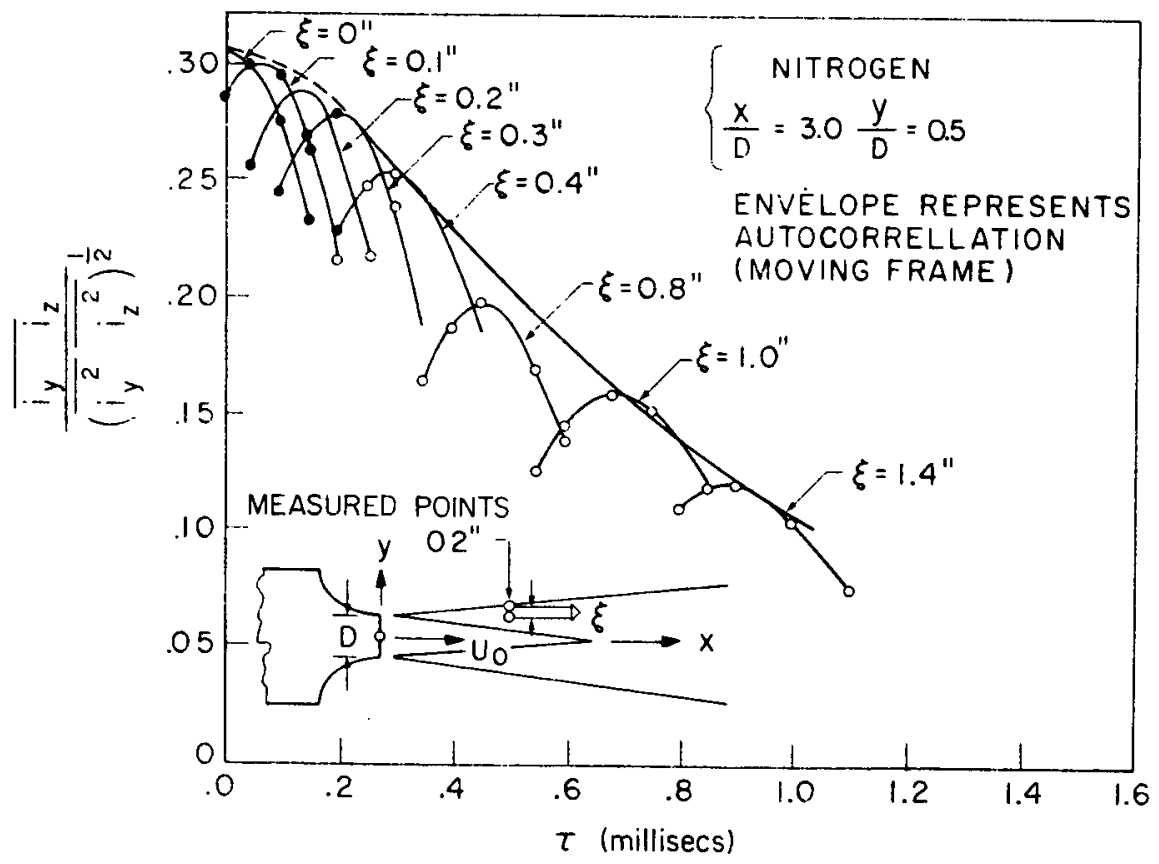

Figure 2. Crossed Beam Correlation in a Subsonic Jet 


\section{A. J. MONTGOMERY}

function obtained for one particular separation of the two beams. The convection velocity can be obtained from any of these curves, with the exception of the one in which $\xi=0$, by dividing the known value of $\xi$ by the time delay corresponding to the peak of the correlation function. The maximum value of the correlation function decreases as the beam separation increases. This behavior reflects the fact that as an eddy moves downstream it gradually loses its identity. The eddy-lifetime is defined as the time in which the envelope decreases to $1 /$ e of its initial value. The space correlation coefficient $r(\xi, 0)$, which can be generated by noting the points at which the individual crosscorrelation curves interest the $=0$ axis, may be integrated over all $\xi$ to obtain the integral scale of the turbulence, and the cross-power spectrum may be obtained by the Fourier transform of the cross-correlation function.

It was noted in the above discussion that the choice of optical wavelength has to be such that there is absorption or scattering along the beam so that turbulence effects therefore result in fluctuations in the detected signal. In supersonic flows, where there are significant variations in air density, some measurements were made at a wavelength of $1850 \mathrm{~A}$ where oxygen absorbs. In subsonic flows, and in some experiments in supersonic flows, a tracer was introduced in the flow, and the fluctuations in visible light produced by variations in the number of tracer particles along the beam were detected.

In the subsonic case very good agreement was obtained with hot wire measurements, and this comparison also demonstrated the high spatial resolution that may be obtained using the crossedbeam technique. No comparison of the supersonic results with previously measured values is possible, since these measurements represent an advance in state of the art.

\section{ATMOSPHERIC MEASUREMENTS}

In the atmosphere the use of artificial light sources would be most undesirable since this would severely limit the application of the technique. Natural, distributed sources fall into three categories

a) Scattered Sun1ight

b) Thermal Emission

c) Non-Thermal Emission (for example, airglow).

Figure 3 shows the atmospheric radiation background for two altitudes. During daylight hours scattered sunlight predominates for wavelengths shorter than approximately 3 microns. At night scattered starlight, scattered moonlight and airglow are the main sources of radiation in the visible and near infrared portion of the spectrum. 

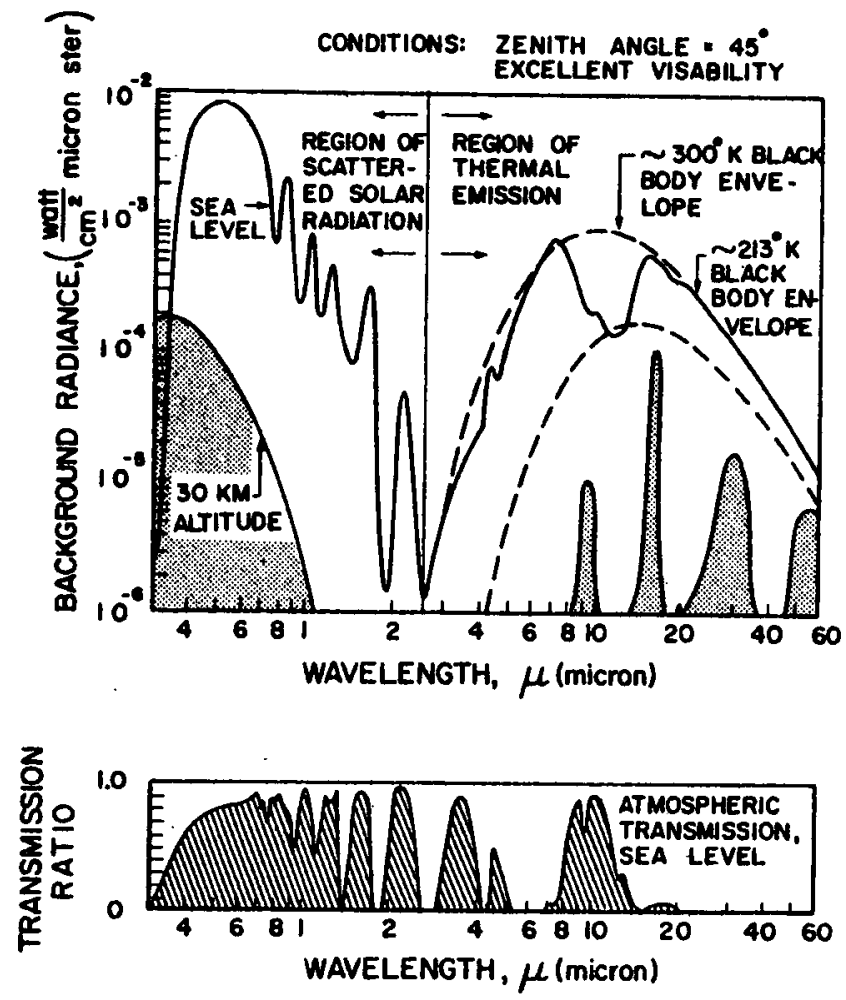

Figure 3. Emission Spectrum of Atmospheric Radiation Background for Two Altitudes.

To apply the crossed-beam technique to atmospheric measurements, the correlated portion of the detected fluctuations signals must be of such a magnitude that it can be pulled out of the noise in a reasonable integration time. The noise signal will consist of uncorrelated fluctuations from parts of the beam away from the intersection point, detector noise and shot noise associated with the mean level of radiation incident upon the detector. Ideally the design of the optical system would ensure that the predominant source of noise is due to atmospheric fluctuations, but if narrow spectral bandwidths have to be used in the infrared portion of the spectrum this may not be possible.

Even if the uncorrelated atmospheric fluctuations are the predominant noise source, it may not be possible to isolate the correlated portion of the signal in a reasonable integration time. The nonstationary nature of atmospheric phenomena limits the integration time, so that, if there are too many eddies along the field-of-view of the detector which significantly contribute to the atmospheric fluctuation level, it may not be possible 'pull out' the correlated signals by the cross-correlation, within times for which stationary conditions can be considered to obtain. For this reason, it is necessary to be selective in the type of measurement that is made so that the number of eddies 
along the detector field-of-view which significantly contribute to the measured fluctuation level is limited.

In fact it would be difficult to choose a measurement wavelength for which there are equal contributions to the detected signal from different altitudes but the contribution from different altitudes is difficult to determine without a more comprehensive knowledge of turbulence scales, and temporal variations in density, aerosol concentration and temperature.

It was shown previously that the covariance of the signals from two detectors with crossed fields-of-view is given by

$$
\mathrm{G}(\mathrm{x}, \mathrm{y}, \mathrm{z})=\left\langle\mathrm{I}_{1}\right\rangle\left\langle\mathrm{I}_{2}\right\rangle \mathrm{k}^{2}(\mathrm{x}, \mathrm{y}, \mathrm{z}, \mathrm{t}) \mathrm{L}_{\mathrm{y}} \mathrm{L}_{\mathrm{z}} .
$$

This expression contains the square of the extinction-coefficient fluctuations and the product of two scale lengths. The magnitude of the extinction coefficient fluctuations will usually be greater near the ground because the air density and the aerosol concentration are greater, and because of the turbulence generated by flow over uneven terrain. On the other hand, the turbulence scales are less so that there will be a compensating effect, and the altitude region which contributes most significantly to the fluctuating signal is not clear-cut. There are obviously some severe problems in reliably computing contributions to the fluctuations from different altitudes, and this point will be clarified in the discussions of particular cases which follow.

\subsection{Scattered Sunlight Measurements}

A photometer on the ground pointing upwards will receive radiation which is scattered into its field-of-view by both air molecules and aerosol particles. The mean signal level recorded will depend on the elevation and azimuth of the fieldof-view of the photometer relative to the angle of the sun and upon the meteorological conditions. Because of the presence of temperature and corresponding density variations in the air which will be convected through the photometer field-of-view by winds, there will be fluctuations superimposed upon the mean photometer output signal. The magnitude of these fluctuations will depend on the scales of these eddies and the absolute magnitude of the air density and/or aerosol density variations. For a constant eddy scale and percentage variation in scatterer concentration, the decrease in air density with height will reduce the molecular scatter contribution to the fluctuation level at the detector by a factor of 10 for eddies at an. altitude of 8 kilometers as compared to fluctuations at ground level. The aerosol fluctuation contribution under similar conditions will fall off much more rapidly because of the much quicker relative decrease in aerosol concentration with altitude. 
Using the turbid atmosphere model of Elterman (1964) the detected signal will be down a factor of 10 at an altitude of 1 kilometer.

However, since the eddy scales increase with altitude, the ability to detect fluctuations at an altitude of 1 kilometer by the cross-correlation of the photometer signals with the experimental arrangement shown in Figure 4 may not be more difficult than if the measurements are made with the beams crossed at an altitude of 50 meters to detect fluctuations at this level. In fact since scattering at low altitudes is dominated by atmospheric aerosols, and the aerosol concentration decreases very quickly with altitude as shown by the aerosol scattering coefficient in Figure 5, we expect the low altitude fluctuations to predominate and, for this reason, the experiments presently being carried out are aimed towards the measurements of winds and turbulence at altitudes up to about 1000 meters by detection of scattered sunlight.

In this discussion I have somewhat glossed over the origin of the fluctuations in scattered sunlight except to say that they are due to fluctuations in concentrations of aerosols and air molecules. Direct sunlight will be scattered by molecules and aerosols in the field-of-view of the detector so if there is a density increase or increase in concentration of aerosols in a volume element of the field-of-view, there will be an incremental increase in the direct scattered signal. However, light scattered into the field-of-view at higher altitudes may be scattered out of the beam and an increase in density or aerosol concentration in a volume element would therefore cause a decrease in detector signal. In addition there will be a contribution from multiple scattering into the 'beam'. The relative values of these different contributions will depend strongly on the atmospheric conditions and the angle of the sun with respect to the direction of view of the photometers.

If the direct scattered sunlight predominates it would be possible to obtain a positive correlation even in the complete absence of fluctuations in scatterer concentration in the region of the beam intersection point. The sunlight reaching this region has traversed a long path through the atmosphere and therefore there will be intensity fluctuations. Similar fluctuations will be present in the scattered sunlight which could result in a significant correlation between the two detected signals. However, when there is a lateral separation between the two beams as is necessary in wind measurement, this effect is minimized.

Midwest Research Institute (St. John, A. D. and Glauz, W. D. 1968) have performed some calculations to determine the relative contributions to the fluctuations in the detected signal. However, multiple scattering was not included, and further work is definitely necessary in this area. 


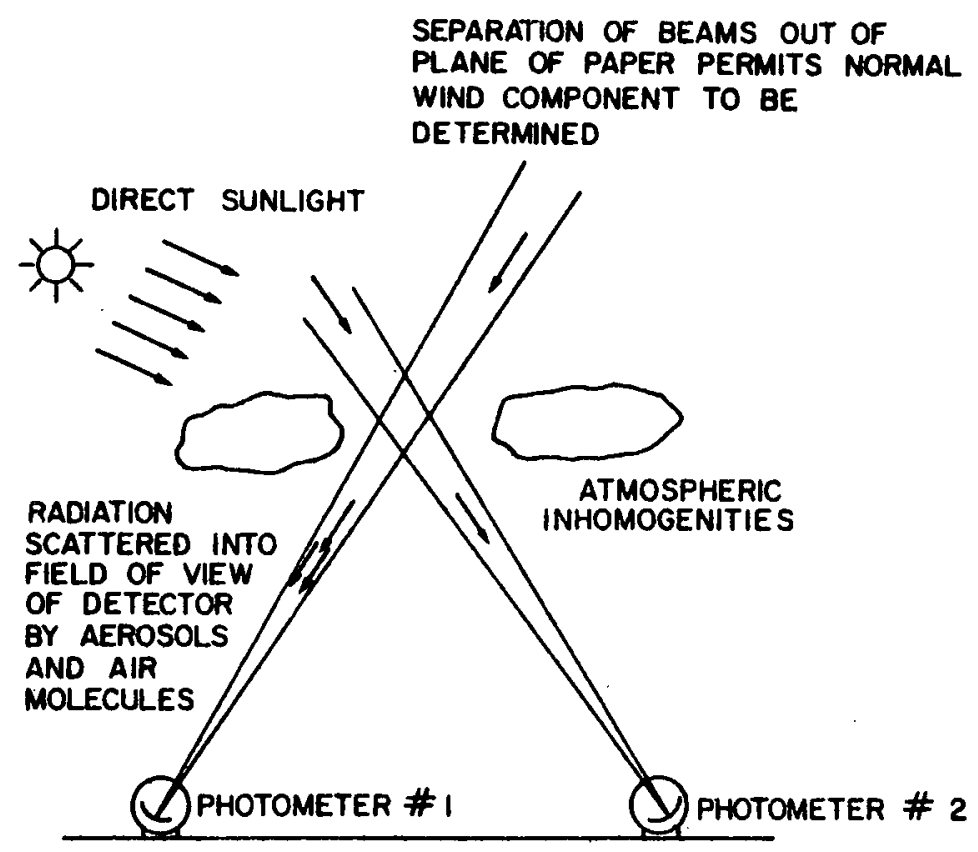

Figure 4. Application of Crossed Beam Technique to Measurement of Atmospheric Winds and Turbulence

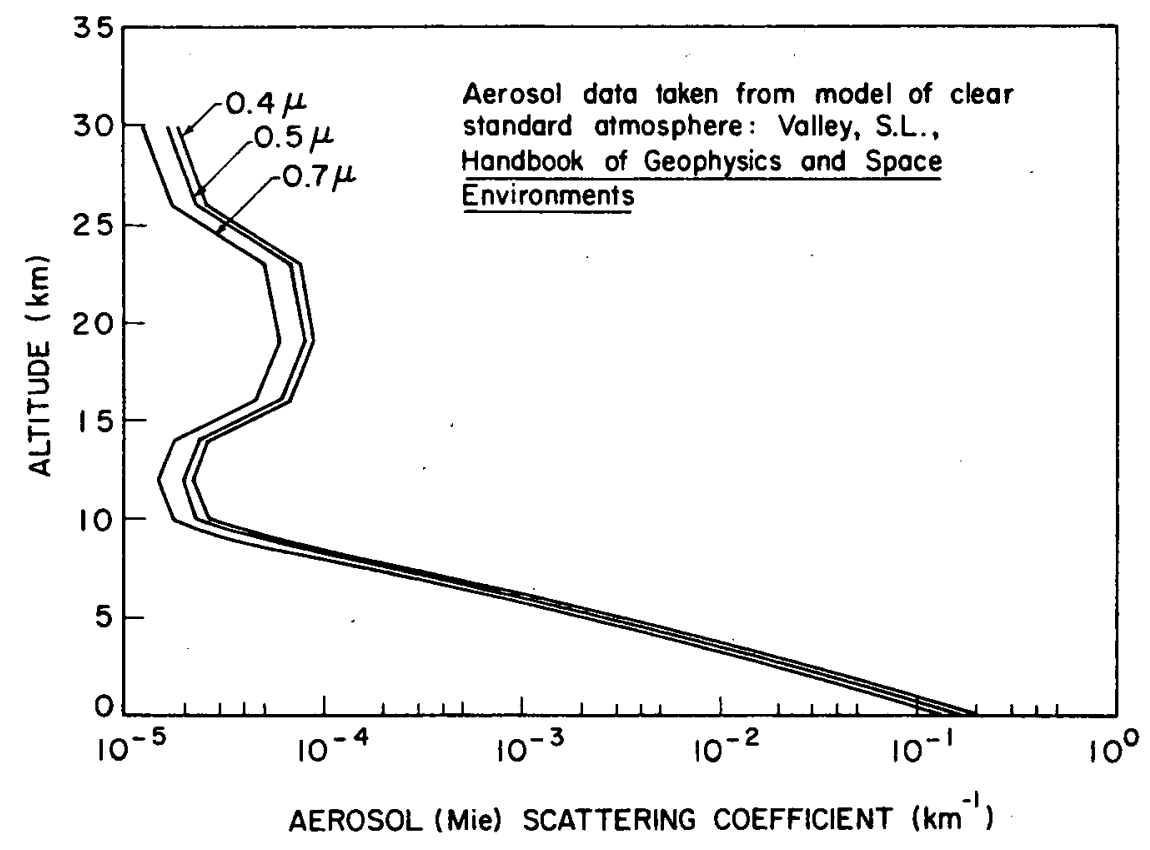

Figure 5. Aerosol (Mie) Scattering Coefficient vs. Altitude for Visible Wavelengths 
REMOTE SENSING OF WINDS AND ATMOSPHERIC TURBULENCE

\subsection{Thermal Emission}

Although successful measurements have been made detecting scattered sunlight in the visible portion of the spectrum, this particular method is restricted to substantially cloudless sky conditions. If an isolated cloud enters the field-of-view of the detecting system there is an increase by a factor of the order of 5 in the detected signal. With a response extending down to 0.01 cycles per second the recorded ac signal will also increase to a leve1 which will be dependent upon the rate of movement of the cloud. When the cloud fills the fieldof-view a main source of signal fluctuations will be the nonuniformities in the cloud itself.

If this signal were recorded the power associated with the fluctuations due to clouds would generally dominate the signals, and spurious cross-correlations would be measured. However, if clouds are in the field-of-view for only a small fraction of the recording time then this effect can be eliminated by setting the signal at zero whenever a cloud is in the fieldof-view of either photometer system.

Potentially these problems can be largely overcome if water vapor is used as a tracer and measurements are made in the vicinity of the water vapor absorption band at 6.3 microns . The exact choice of wavelength will determine the relative signal received from volume elements at different distances from the detecting system. ESSA is presently studying this problem.

Measurements of wind profiles at higher altitudes could be made by detecting the thermal emission of ozone at 9.6 microns. Some preliminary calculations (Krause et a1., 1966) indicate that fluctuations of the order of $0.0 \overline{1 \%} \frac{\text { could be }}{\text { could }}$ detected with 8 inch collector optics and state-of-the-art detectors. We are presently studying these approaches and plan field tests to determine their potential and to further increase the usefulness of the crossed-beam technique as applied to atmospheric measurements.

\section{MEASUREMENTS OF SCATTERED SUNLIGHT FLUCTUATIONS}

The photometer systems used in the atmosphere measurements of scattered sunlight have the following specifications:

Frequency Response

Mean Signal/RMS Noise

Field-of-View

Variable within range $0.01-300 \mathrm{~Hz}$

Spectra1 Response

5-30 minutes of arc

Spectral Bandpass

$0.4-1$ micron

Variable (minimum 500A) 


\section{A. J. MONTGOMERY}

Details of the design of the photometer system are given in the appendix. A photograph of one of the photometers is shown in Figure 6 and the instrumentation van in Figure 7.
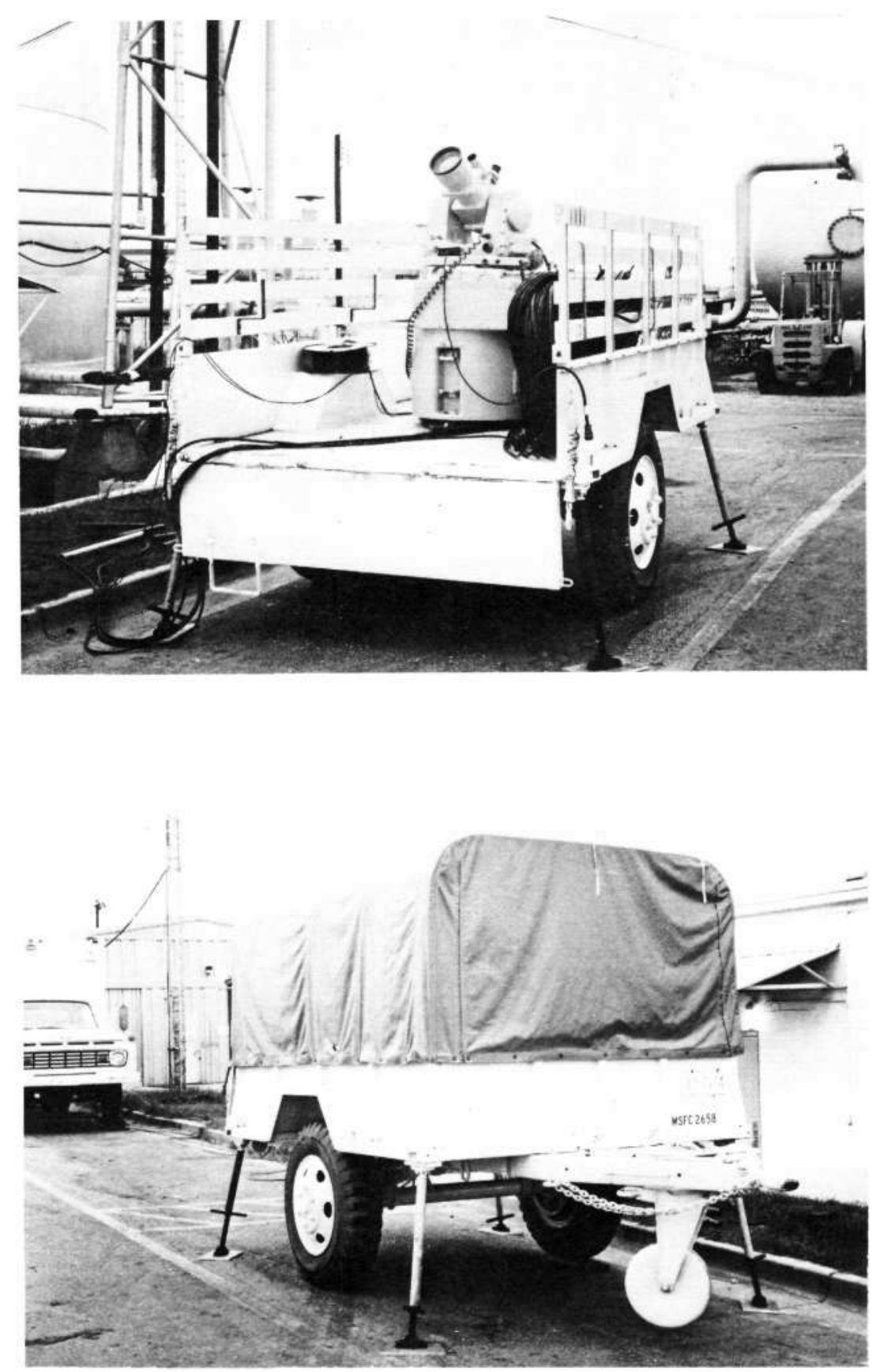

Figure 6. Photometer Trailers (by Courtesy of MSFC) 
REMOTE SENSING OF WINDS AND ATMOSPHERIC TURBULENCE

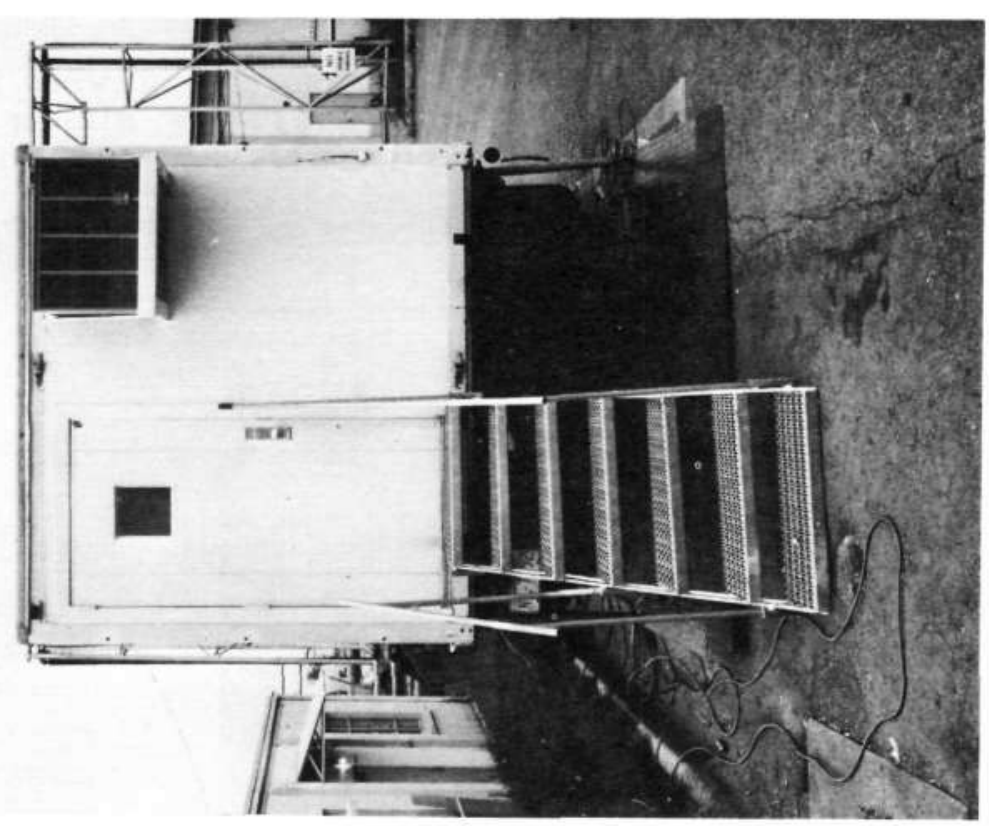

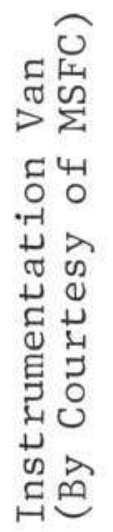

N

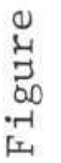
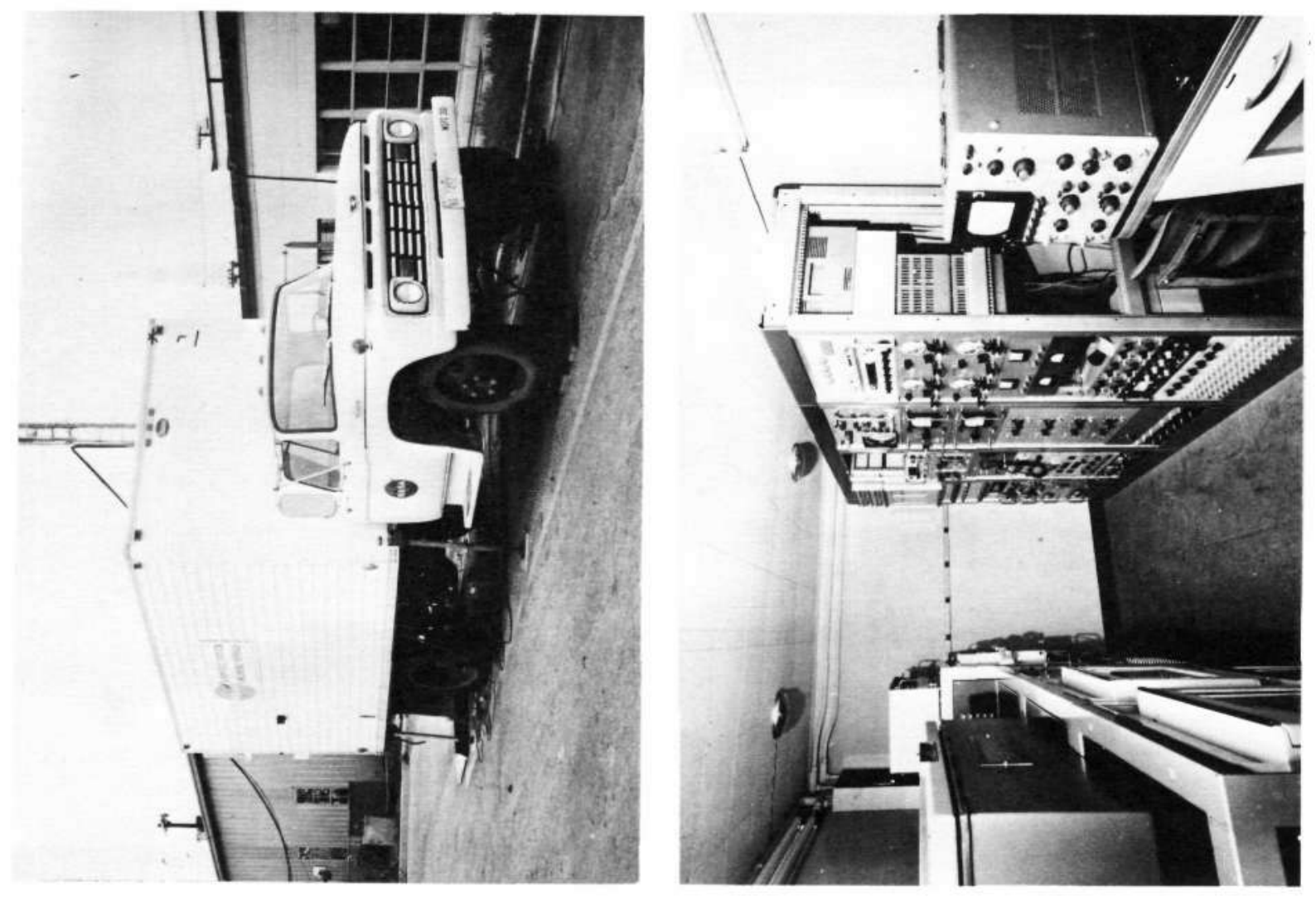
Power spectral density measurements of the fluctuations under clear sky conditions are shown in Figures 8 , and in Figure 9 these results are replotted with the ordinate - power spectral density $x$ frequency. In this second plot the area underneath the curve in a particular frequency interval, $\Delta \mathrm{f}$, is directly proportional to the energy in this frequency interval. These results were obtained by playing back the recorded signals through a variable bandpass filter and measuring the output level with a RMS voltmeter. It can be seen that the fluctuations follow quite closely a -6.5 power law down to frequencies less than 0.1 cycles per second. The roll off beyond 0.01 cycles per second is due in part to the high pass filter which reduces the amplitude of the signal $3 \mathrm{db}$ at 0.01 cycles per second and winds down at the rate of $6 \mathrm{db}$ per octave.

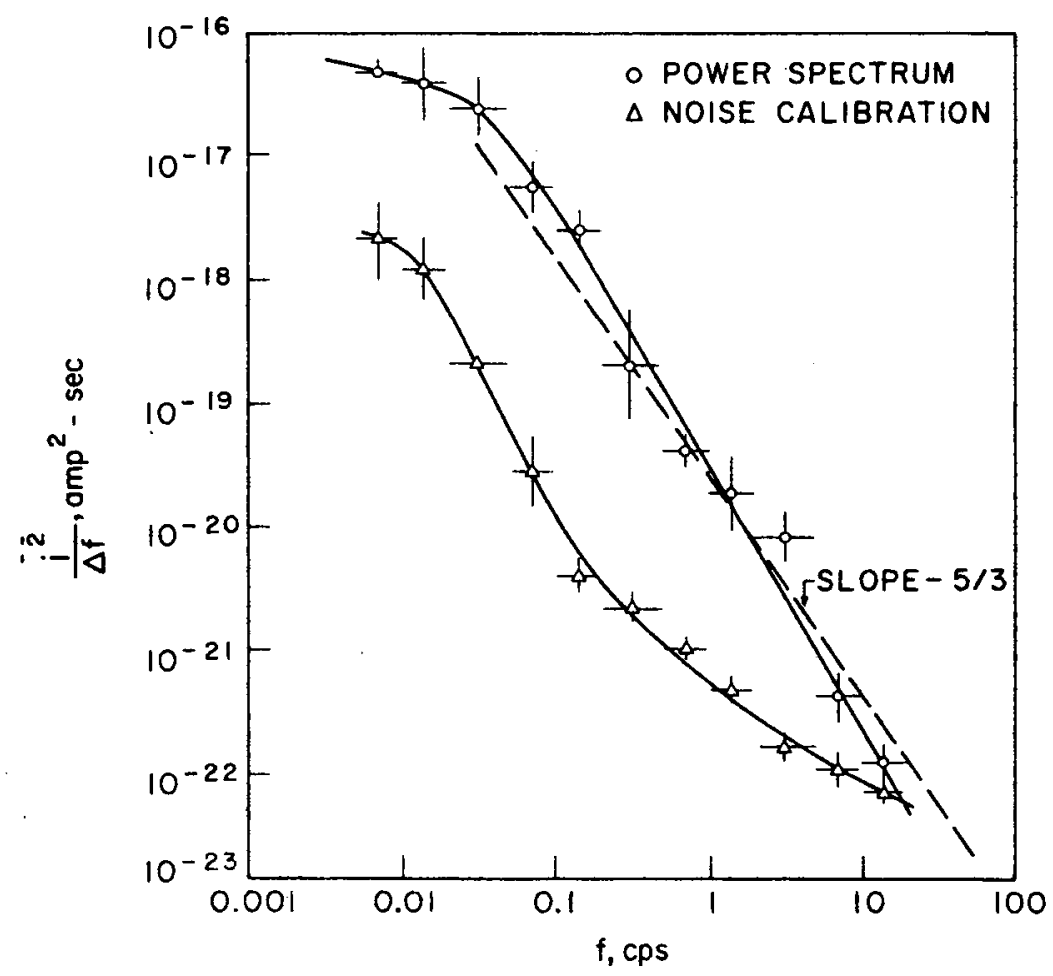

Figure 8. Single Beam Power Spectrum $\frac{\overline{i^{2}}}{\Delta f}$ vs. f 


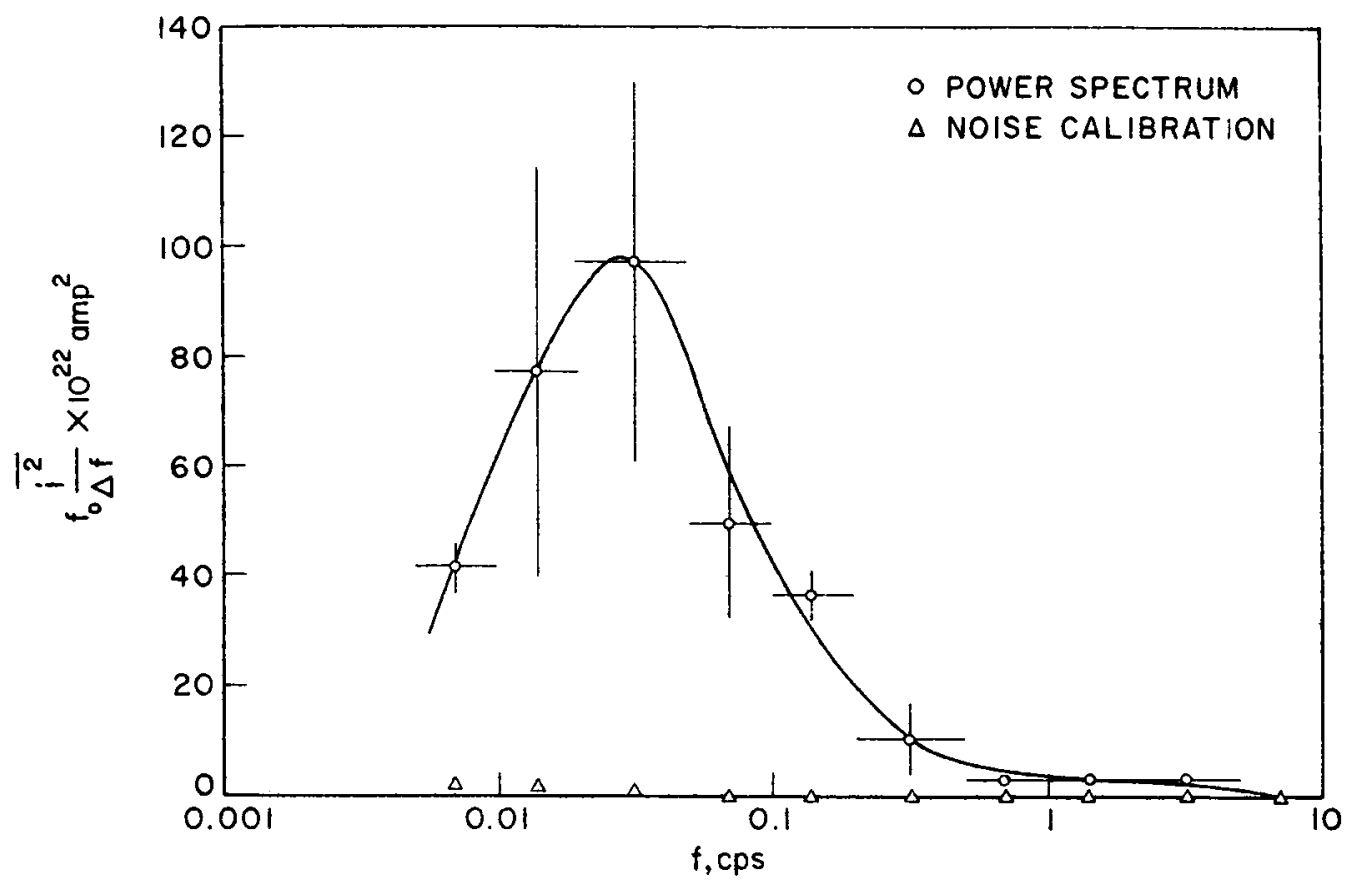

Figure 9. Single-Beam Power Spectrum, $\mathrm{f}_{\mathrm{o}} \frac{\overline{i^{2}}}{\Delta \mathrm{f}}$ vs. $f$

A line of slope $-5 / 3$ is also shown in Figure 8 and it is evident that the results differ significantly from the $-5 / 3$ dependence that would be expected in point measurements. This is not an unexpected result because of the integration along the line of sight. The contribution of an eddy, being convected through the field-of-view of the photometer system, to the fluctuating signal will be proportional to both the variation in the number of scatterers associated with the eddy, and the size of the eddy measured along the field-of-view of the detecting system. Because the contributions from different eddies are uncorrelated then the resulting rms fluctuation from $n$ eddies along the line of sight will only be $n$ times the contribution of a single eddy. This intuitive argument would indicate that a $-8 / 3$ power law might be expected. At this time 
there is no proven explanation of the $-6.5 / 3$ power law that is actually measured, however, if the turbulent eddies were elongated in the flow direction, such a reduction in the power dependance could be observed. The effect of the increase in eddy scales and the decrease in the magnitude of the fluctuations with altitude would also be expected to change the power spectrum, but no attempt has yet been made to study the way in which these variables would effect it.

\section{CROSSED-BEAM MEASUREMENTS OF WINDS}

Since atmospheric crossed-beam measurements detecting scattered sunlight are continuing at the present time this paper is a status report of the results obtained to date. Successful wind measurements have been made, and this success has gone hand in hand with the development of suitable statistical methods to reduce the effects of non-stationarity in the data.

The geometry of the crossed-beam arrangement used in the atmospheric tests is shown in Figure 10. The location and

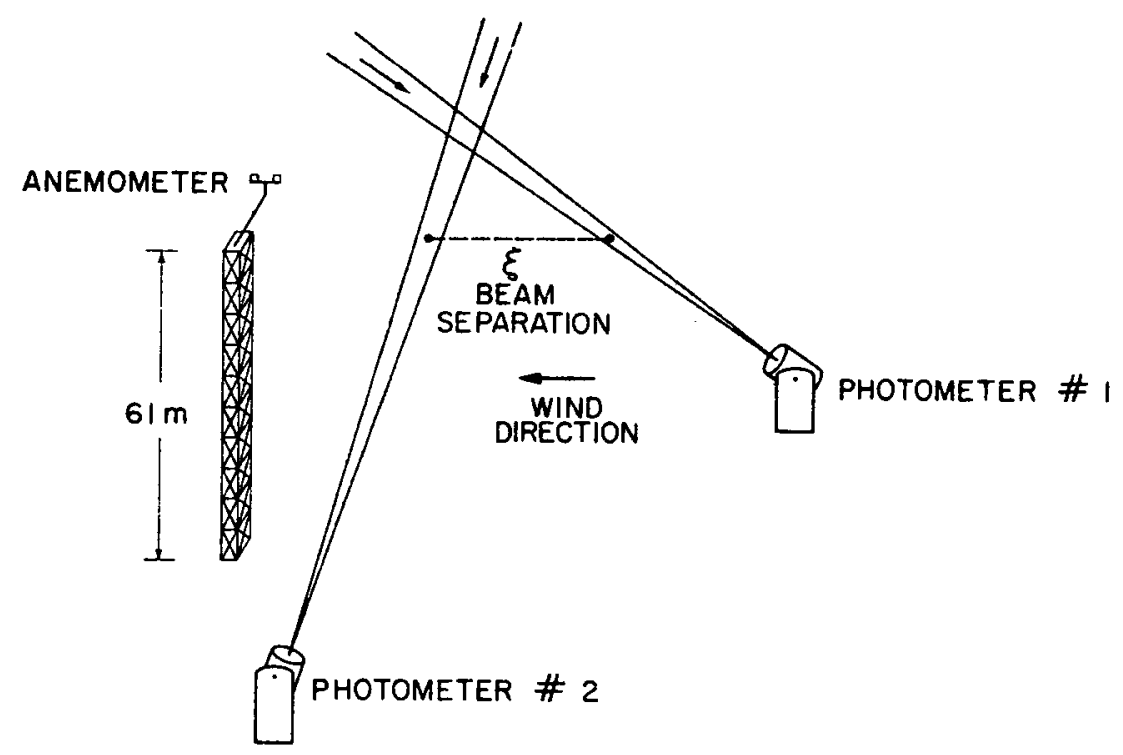

Figure 10. Geometry at Atmospheric Crossed Beam Arrangement. 
viewing direction of the photometers are usually made to lie in two parallel vertical planes which are chosen to be approximately perpendicular to the wind direction. Most of the measurements to date have been made with the two beams crossed at an altitude corresponding to the top of the meteorological tower. Thus a direct comparison between the wind measured with an anemometer on top of the tower and that obtained from the crossed-beam measurements is possible. It should be noted that the altitude at which the wind is in fact measured depends on the wind direction. If the wind direction is perpendicular to the parallel vertical planes containing the lines of sight of the detector systems, then the altitude of measurement will be the height at which the beams would intersect if the two vertical planes were coincident. For all other wind directions an eddy can only traverse both 'beams' at a different altitude determined by the angle between the beams and the wind direction. Since in a practical case both the wind speed and the wind direction will be varying, the result obtained with the crossed-beam system represents a complex type of averaging over both altitude and time. If the wind direction is fairly constant within $+20^{\circ}$ of the plane of the beams then the altitude region over which the averaging will take place will be small, and the crossed-beam measurement $c$ an be considered to yield an average wind velocity at the crossing altitude.

Before describing some of the experimental results that have been obtained some comments about the fluctuation records, and the data processing are in order. Under clear skies the signal fluctuations with a bandpass from $0.01-10 \mathrm{~Hz}$ are typically of the order of $1 \%$ of the mean signal level. Although this fact is not completely established, the fluctuation levels appear to be less after a rain when the ground is still damp. This is not at all unlikely since the aerosol concentrations near the ground would be lower under these circumstances. In a 45 minute record it is usually found that there are portions of the record in which the fluctuation level is considerably higher than the average. Sometimes there is an obvious cause for this, such as a car going by on a nearby dirt road raising a considerable dust cloud which drifts through the detector field-of-view. At other times there is no apparent reason for the increased activity. In any case, if a straight averaging procedure were used in the data processing, the portions of the record in which the fluctuations are large tend to dominate the correlation results.

To avoid this problem the data are processed in the computer in 6 minute segments, and the time averaged cross product is normalized with respect to the rms levels in that segment of the record before the pieces are averaged through the entire record. In other words, the correlation function is found for each segment and this procedure successfully prevents any particular piece from dominating the entire record.

Despite these precautions, spurious correlations are still sometimes obtained which make identification of the peak in the 


\section{A. J. MONTGOMERY}

correlation function corresponding to the wind speed ambiguous. The so-called statistical error is utilized to help solve this problem. In simple terms a correlation function is found for each six minute piece of data throughout the record. These correlation functions are averaged and the rms variation in the value of the correlation function is found for each time delay. It is this rms variation, suitably weighted to obtain the desired statistical certainty in the result, which is called the statistical error.

Ideally, if a stationary situation existed, the statistical error would decrease as the square root of the number of pieces included; however, it is found in the atmospheric data that the error begins to decrease, but after some time period (usually between 30 minutes and one hour) the error either oscillates or starts to increase again. The integration time is therefore chosen as the time in which the statistical error reaches its minimum value.

Figure (11-14) shows some of the results obtained which have been previously presented by Stephens, Sandborn and Montgomery.

Figure 11 is the result for a zero separation case with the fields-of-
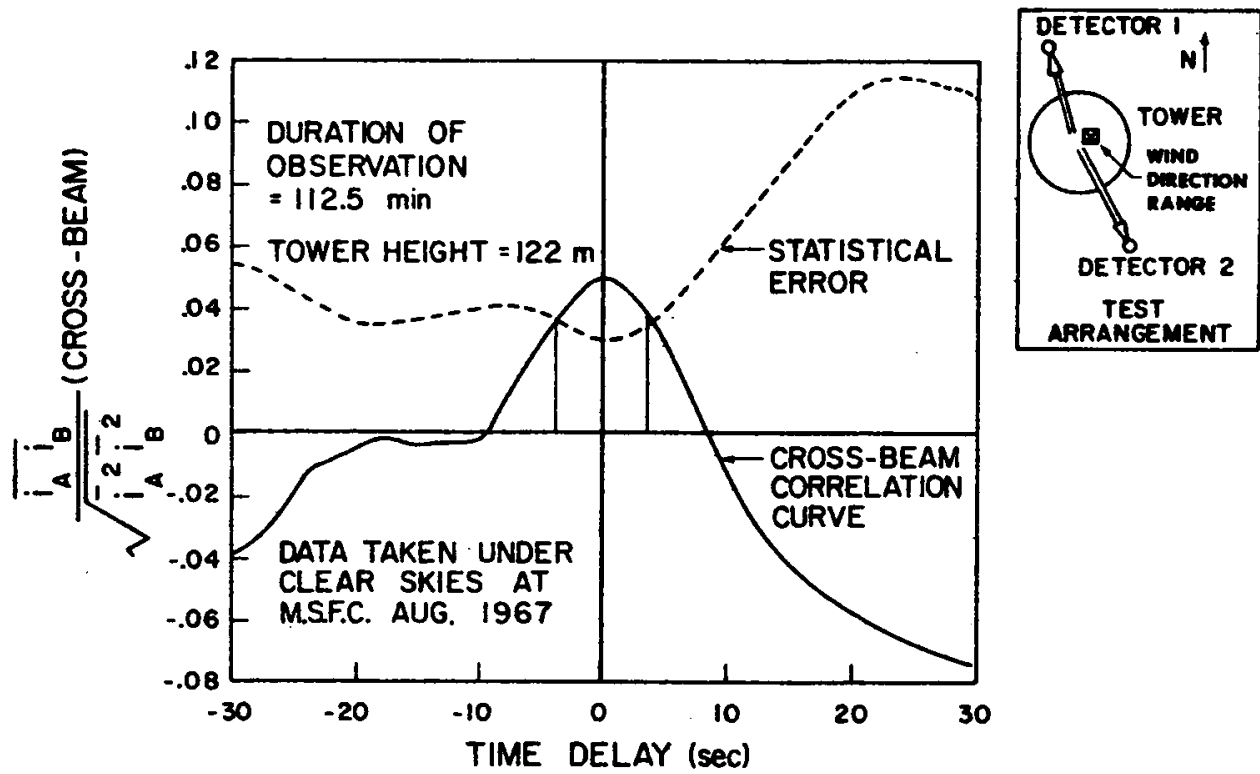

Figure 11. Cross Correlation Function - Zero Beam Separation 
view of the two detectors intersecting at an altitude corresponding to the top of the meteorological tower at MSFC. No wind information is of course available from this measurement. The correlation coefficient is quite smal1 0.05 and the peak only slightly exceeds the statistical error. However, the peak does occur accurately at $t=0$ as would be expected in this measurement. The statistical error is associated, of course, with the value of the correlation function; hence with a $90 \%$ probability the correlation function at zero time delay is $0.05+0.03$.

This statistical probability results from the choice of weighing factor applied to the rms variations in the correlation function. Plotting the results in this manner is used for ease of presentation.

The remaining wind measurements were made at the Colorado State University meteorological test site in the North Platte River Valley, located in northeastern Colorado, approximately fifteen miles east of the first major pressure rise of the Rocky Mountains.

Figure 12 shows the anemometer data plotted as a first order probability density of velocity, and the correlation function plotted in terms of a wind velocity. Since the beam separation was 30 meters the long tail of the cross-correlation curve

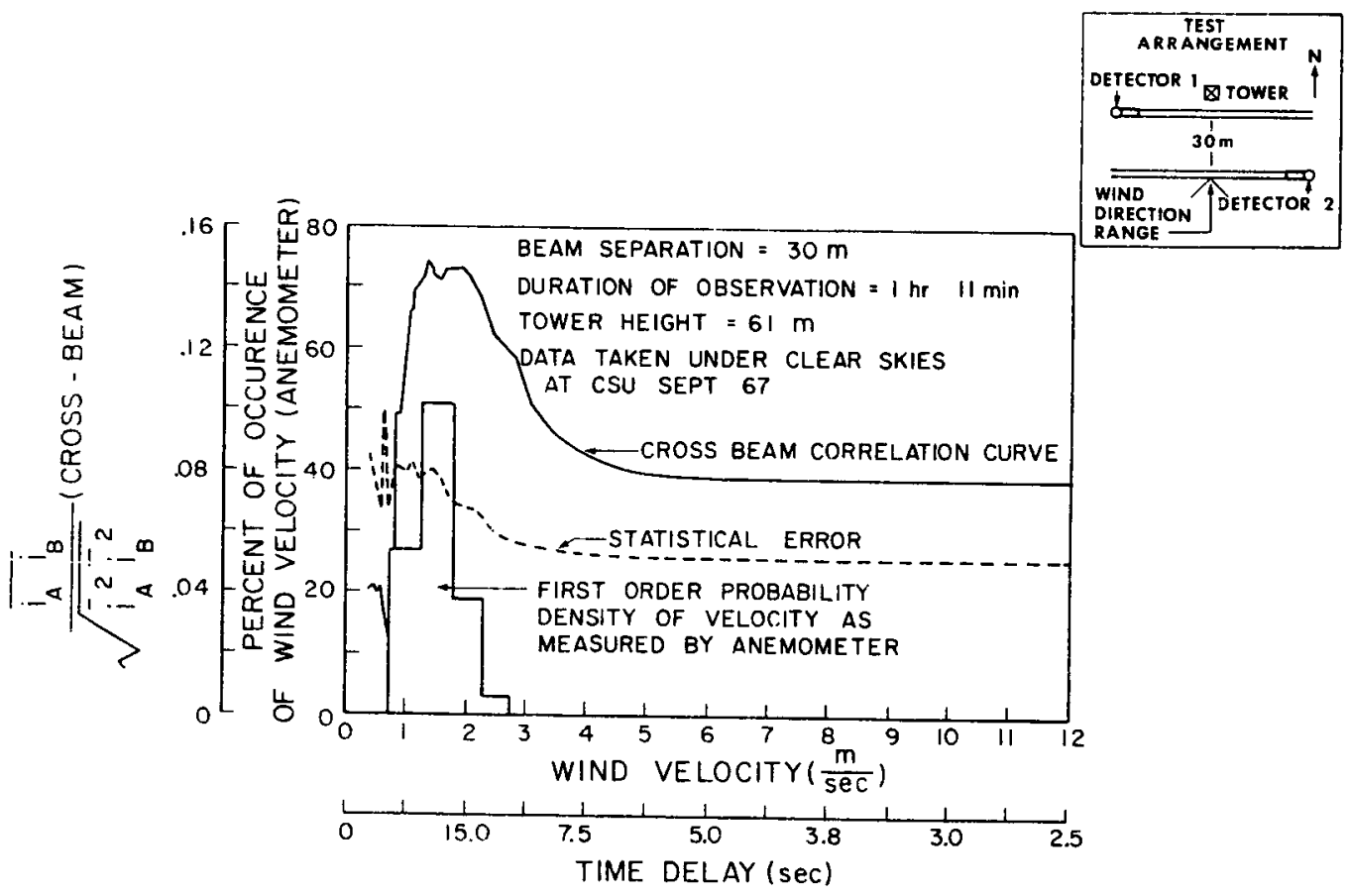

Figure 12. Light Wind Case $\sim 3 \mathrm{mph}$. 
represents delay times from 2.5 to 5 seconds as compared with the 5-30 second time delay range covered by peak. Thus this tail results from the method of presentation and is not significant. There is quite good agreement between the anemometer and the crossed-beam results although the winds were light and variable.

Higher wind cases are shown in Figure 13 and 14 in which, once again, good agreement is shown between the anemometer and the crossed-beam results. In Figure 13 the statistical error is relatively small and the correlation coefficient at the peak is large. Under these windy conditions a greater concentration of aerosols might be expected to be present close to the ground, and this could weight the results by increasing the contribution to the fluctuating signal from low altitudes. This would have the effect of decreasing the number of eddies along the fieldof-view of the photometers which contribute significantly to the fluctuation level, and therefore increase the correlation coefficient.

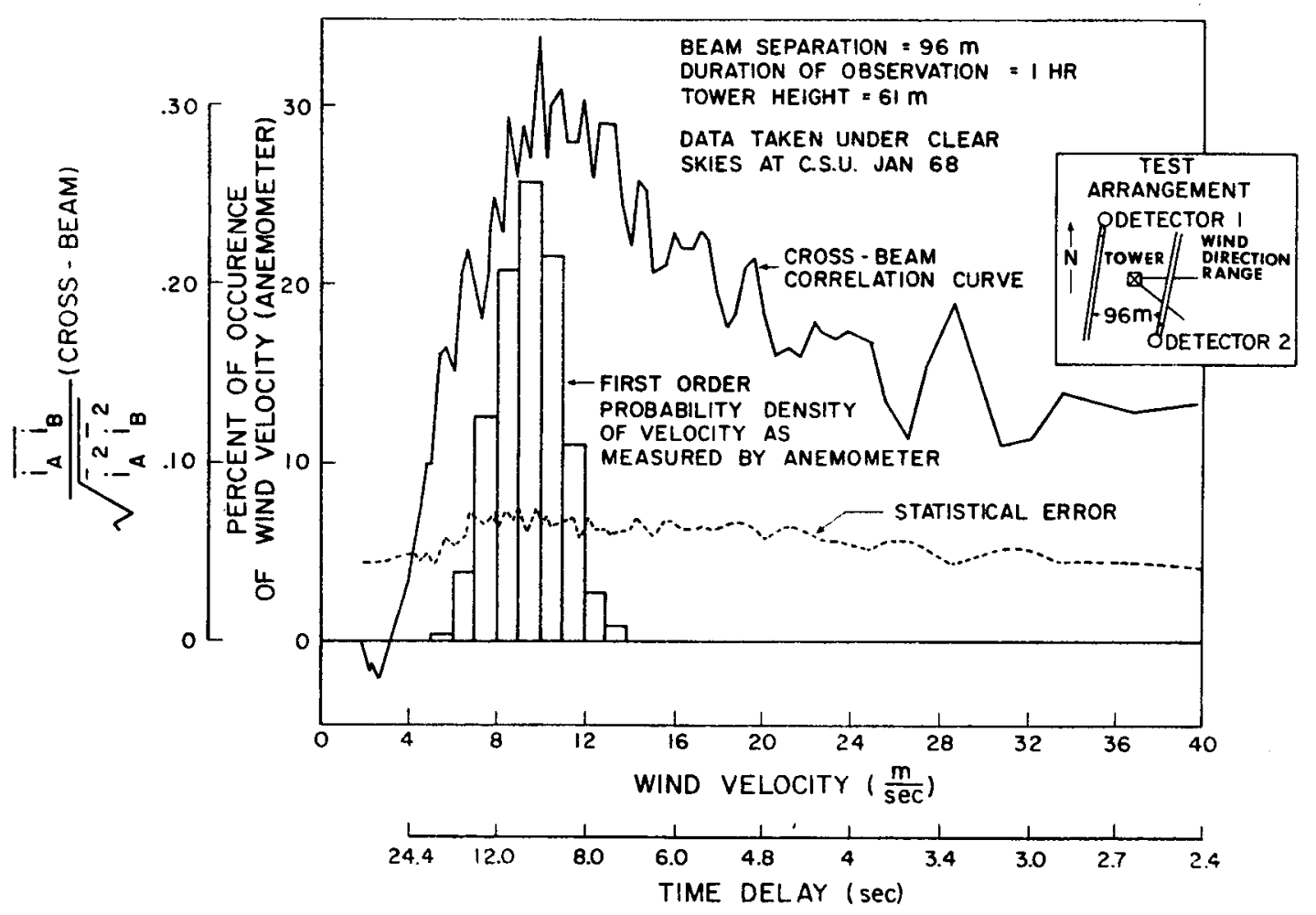

Figure 13. Medium Wind Case $20 \mathrm{mph}$. 


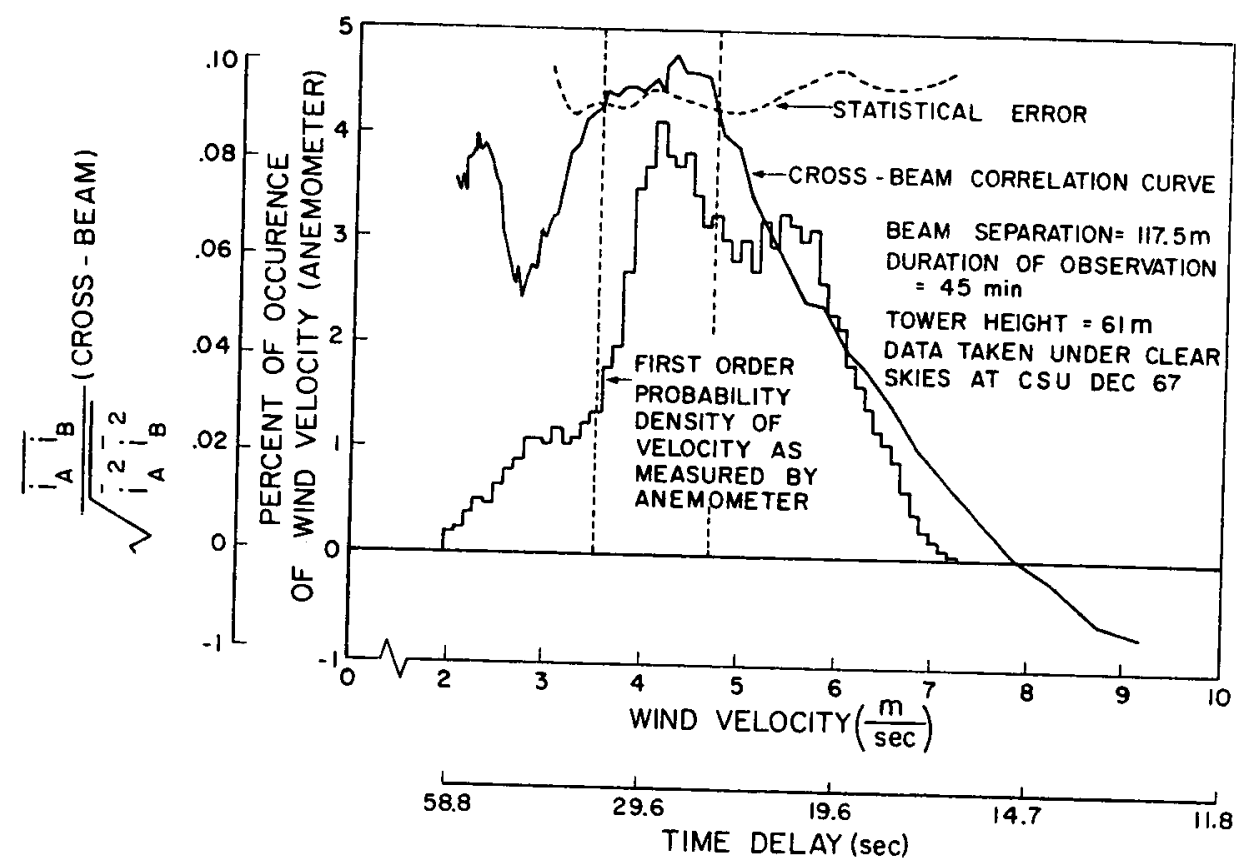

Figure 14. Low Wind - Large Beam Separation Case.

The results described above are summarized in the following table. There is encouragingly good agreement between the crossedbeam and the anemometer results. The field tests are continuing show the same promise of the results described in this paper.

\section{FUTURE DEVELOPMENT OF THE CROSSED-BEAM TECHNIQUE}

The immediate future developments of the crossed-beam technique have to be directed towards exploring the potential and the limitations of the method. For example, in order to remove some of the ambiguities which sometimes arise in determining the peak in the correlation function which corresponds to the wind speed, a multiple beam system is being developed. This will permit results for several beam separations to be obtained simultaneously, and in addition, because the beam will perforce be in a fan configuration, the wind direction can also be found if the correlation maxima are sufficiently well defined. 


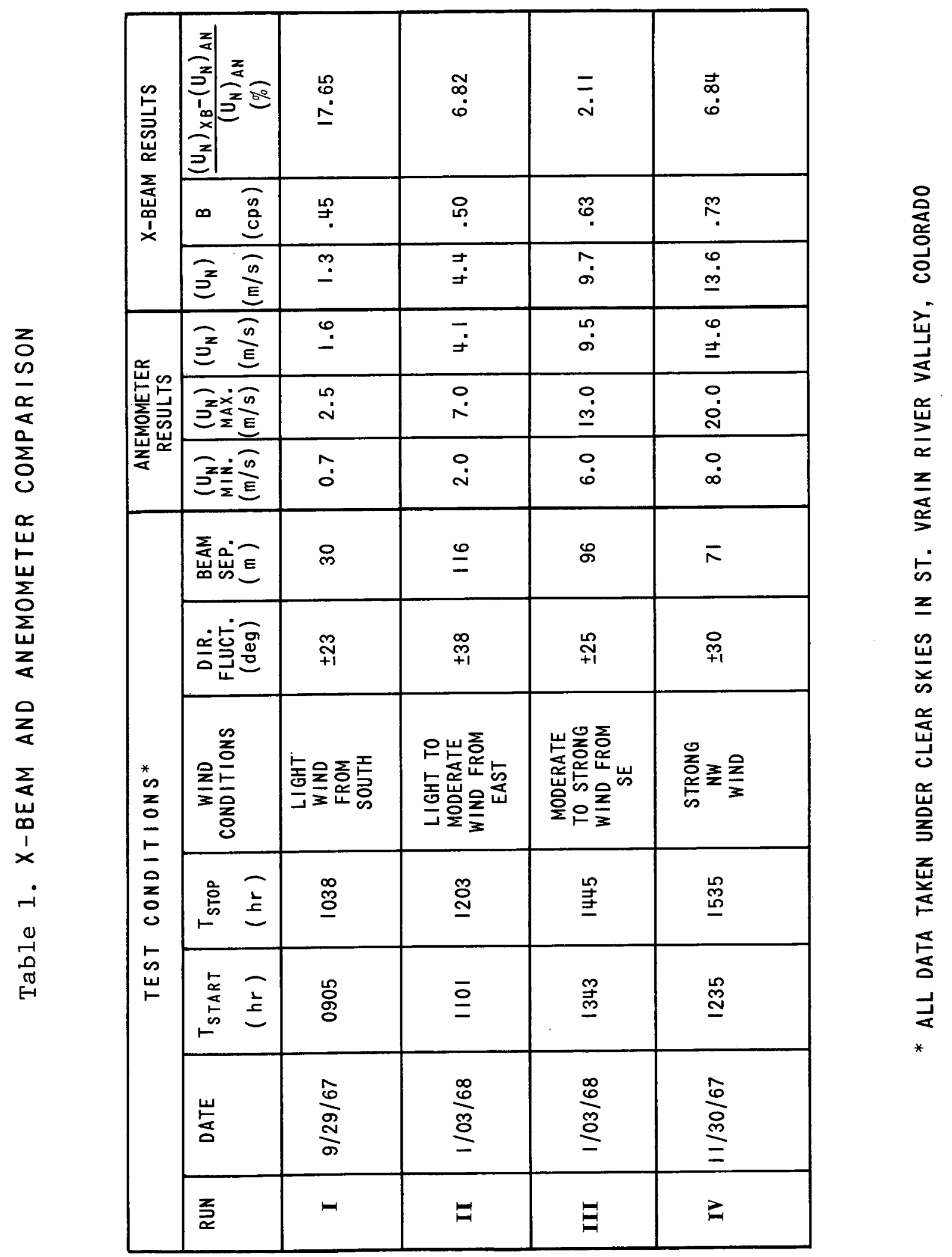


To avoid the limitations imposed on the present system under the cloudy skies the potential of infrared measurements has to be explored. The physics of the problem in terms of trying to define the altitude contributions to the fluctuating signals in both the scattered sunlight and infrared cases has to be studied. This would permit estimates to be made of the altitude resolution and maximum altitudes for which useful wind data may be obtained.

Some new approaches in terms of the data reduction methods have to be developed. The use of the derivatives of the signals to effectively flatten the frequency spectra and narrow the correlation peak is presently being tried. Further examination of the statistical error, and the relative errors between correlations for different time delays obtained from the same data record have to be examined.

\section{CONCLUSIONS}

The potential future development of the crossed-beam technique once the feasibility of its application to atmospheric measurements has been demonstrated is very great. There are a large number of different types of measurements, some of which have been discussed above, that can usefully be made which will contribute to the understanding of atmospheric phenomena, and to improvement in weather forcasting.

Successful ground tests should be followed by measurements from aircraft. Clear air turbulence is of great importance as far as its effect on aircraft operations is concerned, and much could be learned of this phenomena by crossed-beam measurements from an aircraft. Of course in the aircraft case, instead of the fluctuations being produced by turbulence eddies being convected through stationary beams, the fluctuations will be largely produced by the movement of the "beams" or photometer field's of view through regions of atmospheric turbulence. The fluctuation frequencies will be considerably different. Also in the aircraft case, time delay tricks as illustrated in Figure 15 may remove the necessity of having two photometers separated
in space.

The two photometers are mounted on a stabilized platform and have an angle $\theta$ between their respective fields of view. If the turbulence structure is assumed to be frozen, then the fluctuations detected by photometer 非 originating from a height h above the aircraft will be repeated in signal from photometer \#2 a time $\tau$ later, where $\tau$ is the distance d divided by the velocity of the aircraft. The altitude region of interest can therefore be studied by selecting the appropriate time interval $\tau$. To obtain wind speeds it is necessary to separate the photometer fields of view in a direction perpendicular to the plane of the 


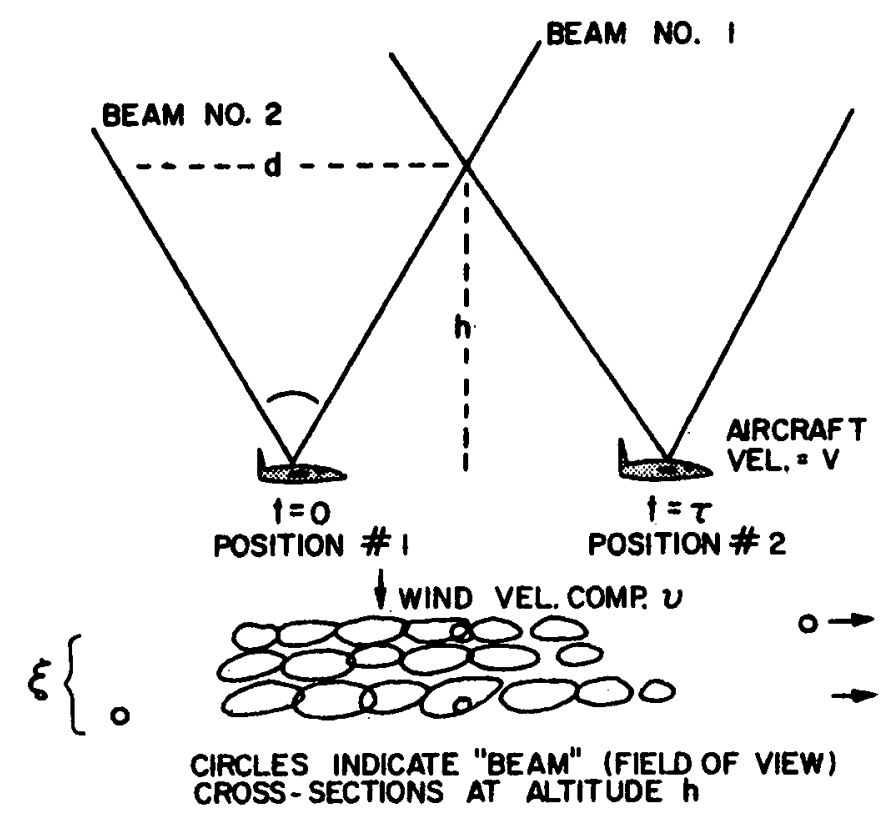

Figure 15. Scanning System Concept - For Particular Wind Speed $\mathrm{u}$ and Beam Separation $\xi$ We have Time $\tau$ for Line of Eddies Originally in Beam 1 作 to Reach Beam 非2. At Height, $h$, Beam Delay is $\tau$, so a Maximum Correlation is Recorded For This Time Delay. With a Fan of Beam Giving Number of Different $\xi^{\prime}$ s the Wind Component as a Function of Altitude May Be Measured.

paper. An ambiguity is introduced here which can only be resolved by use of a fan of "beams" from one of the photometer systems. This problem has been discussed by Krause et a1. (1966) and by Krause (1967) and will not therefore be considered further here.

The logical development of the technique through an aircraft operation phase together with the study of spectral regions in which signals are detected and which could be utilized by downward looking systems, would then enable the potential of satellite experiments to be accurately assessed. 


\section{ACKNOWLEDGEMENTS}

In preparing this review paper I have drawn heavily on the work of my colleagues at IIT Research Institute, and friends and associates at Marshall Space Flight Center and Colorado State University.

The overal1 effort has been funded by NASA, George C. Marshall space F1ight Center. Dr. Fritz Krause is the NASA project monitor and he has made many significant technical contributions to this work.

The atmospheric crossed-beam instrumentation has been fabricated by IIT Research Institute while the recording and ancillary instrumentation has been supplied by MSFC. Check out and the first crossed-beam measurements were made by IIT Research Institute at Marsha11 Space Flight Center, and Colorado State University has been responsible for the field test program carried out in Colorado. A11 the digital data reduction has been done on MSFC computers.

It is not possible to acknowledge all the people who have made significant contributions to this program, however, particular mention should be made of $M$. J. Fisher and $E$. H. Klugman at IIT Research Institute, F. R. Krause and J. Heaman, R. R. Jayroe and J. B. Stephens at MSFC and V. Sandborn at Colorado State University.

\section{REFERENCES}

Elterman, L., (1964), "Atmospheric Attenuation Model, 1964 , in the Uitraviolet, the Visible and the Infrated Windows for Altitudes to $50 \mathrm{~km}, "$ Environmental Research Paper No. 46, AFCRL.

Fisher, M. J., (1964), "Measurement of Local Density Fluctuations in a Turbulent Shear Layer," IITRI Project Suggestion No. 64-107NX.

Fisher, M. J. and Krause, F. R., (1967), "The Crossed-Beam Correlation Technique," J. Fluid Mech. 28, 705-717.

Krause, F. R., Hu, S. S . and Montgomery, A. J., (1966), "On Cross-Beam Monitoring of Atmospheric Winds and Turbulence with Two Orbiting Telescopes," NASA TMX-53538. 
Krause, F. R., (1967), "A Passive Optical Technique for Remote Sensing of Horizontal Wind Profiles and Atmospheric Turbulence," Presented at Forty-eighth Annual Meeting of the American Geophysical Union.

St. John, A. D. and Glauz, W. D., (1968), "Study of Atmospheric and AAP Objectives of Cross-Beam Experiments," NASA Contractor Report NASA CR-61191.

Stephens, J. B., Sandborn, V., Montgomery, A. J. "Remote Wind Detection With the Cross Beam Method at Tower Heights", Presented at the Third National Conference on Aerospace Meteorology May 6-9 (1968). New Orleans, La.

\section{APPENDIX}

DESIGN OF PHOTOMETERS FOR SCATTERED SUNLIGHT MEASUREMENTS

Optical Design -- The optical design of photometer system was based on the following specifications:

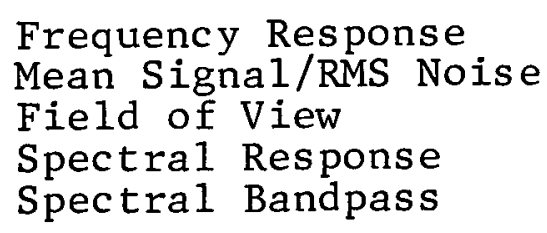

Frequency Response

Mean Signal/RMS Noise

Spectral Response

Spectral Bandpass
$0.001-300 \mathrm{cps}$
104
$5-30$ minutes of arc
$0 . \overline{4}-1$ micron

Variable (minimum 500A)

The system is shown schematically in Figure Al. The objective

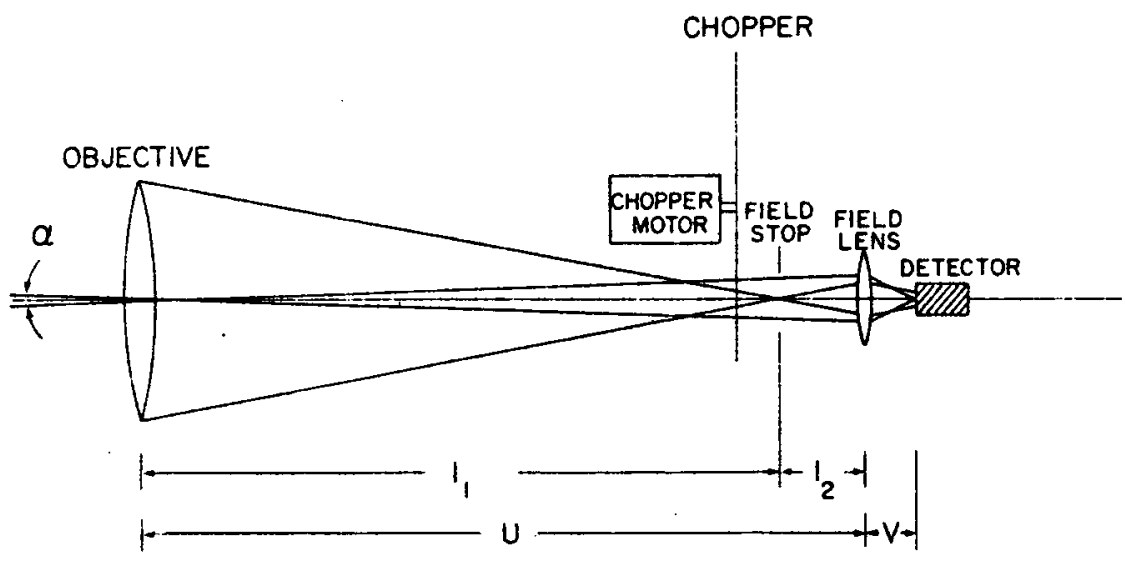

Figure A1. Optical System Schematic 
(collector) lens has an aperture $17.5 \mathrm{~cm}$ and a focal length of $47.4 \mathrm{~cm}$. The field stop which defines the field of view of the photometer is located in the focal plane of the collector lens, and is preceded by the chopper. To avoid any possible problems of nonuniform fields combined with variations in sensitivity over the detector area, a field lens is included which images the exit pupil of the objective on to the detector.

The chopper which has square blades is rotated by its motor approximately $3600 \mathrm{rpm}$ and with 20 blades produces a signal frequency of $1200 \mathrm{cps}$. Use of such a chopper prevents the $1 / \mathrm{f}$ noise of the silicon diode detector or of the preamplifier being the dominant noise source at low frequencies. It also prevents slow drifts, due for example to temperature changes, from being misinterpreted as a low frequency signal component.

Because the intensity of the radiation reaching the detector is relatively high, advantage could be taken of the much greater quantum efficiency available with a silicon diode as compared to a vacuum phototube or photodiode. The detector used is an EG\&G SGD-444 silicon photodiode the quantum efficiency of which is compared to common photoemissive surfaces in Figure A2.

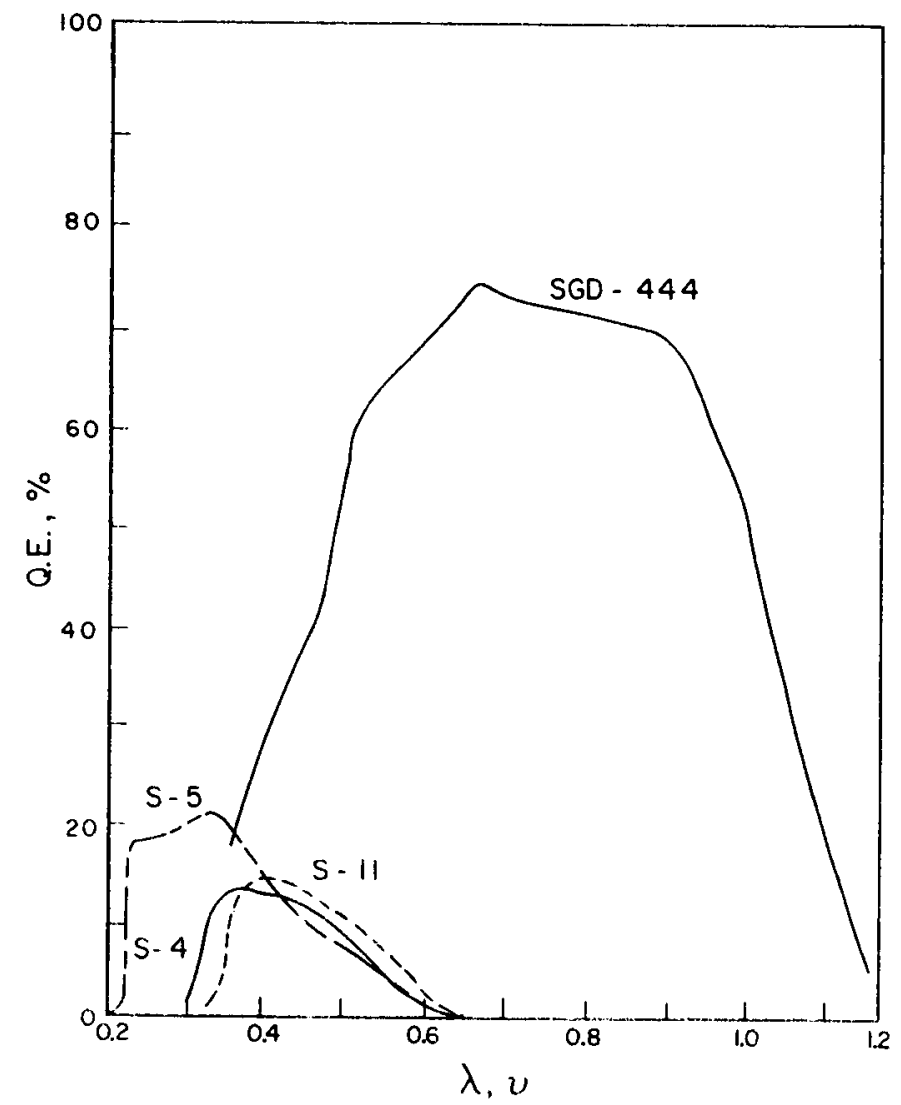

Figure A2. Comparison of Quantum Efficiencies of Severa1 Photodetectors 
Electronic Signal.Processing--The signal processing electronics are shown in Figure A3. The signal from the detector is preamplified before being demodulated and the AC part of the demodulated signal is filtered and amplified. With the low-pass and high-pass filters in their zero position the passband is from 0.01 - $300 \mathrm{cps}$. Both the a.c. and the d.c. signals are recorded on magnetic tape using an Ampex CP100 tape recorder.

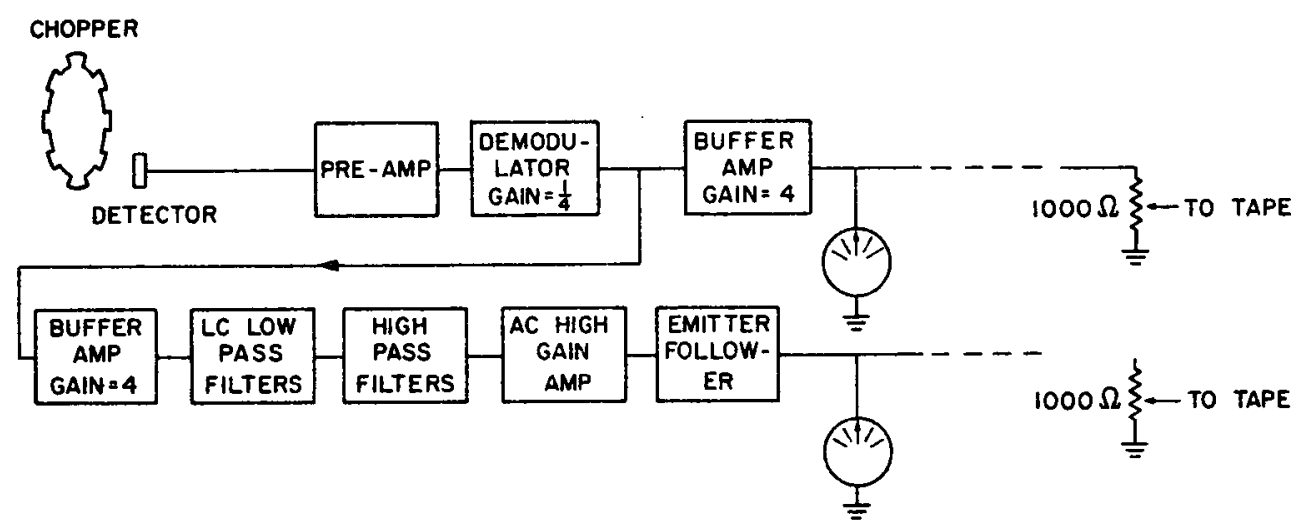

Figure A3. Block Diagram of Signal Processing Chain. 